\title{
Photometry of the SW Sextantis-type nova-like BH Lyncis in high state ${ }^{\star}$
}

\author{
V. Stanishev ${ }^{1,2}$, Z. Kraicheva ${ }^{2}$, and V. Genkov ${ }^{2}$ \\ 1 Department of Physics, Stockholm University, Albanova University Center, 10691 Stockholm, Sweden \\ 2 Institute of Astronomy, Bulgarian Academy of Sciences, 72 Tsarighradsko Shousse Blvd., 1784 Sofia, Bulgaria \\ e-mail: vall@physto.se, [vall;zk;vgenkov] @astro.bas.bg
}

Received 16 November 2005 / Accepted 2 March 2006

ABSTRACT

\begin{abstract}
Aims. We present a photometric study of the deeply eclipsing SW Sex-type nova-like cataclysmic variable star BH Lyn. Methods. Time-resolved $V$-band CCD photometry was obtained for seven nights between 1999 and 2004.

Results. We determined 11 new eclipse timings of BH Lyn and derived a refined orbital ephemeris with an orbital period of 0 d $155875577(14)$. During the observations, BH Lyn was in high-state with $V \simeq 15.5 \mathrm{mag}$. The star presents $\sim 1.5$ mag deep eclipses with mean full-width at half-flux of $0.0683( \pm 0.0054) P_{\text {orb }}$. The eclipse shape is highly variable, even changing form cycle to cycle. This is most likely due to accretion disc surface brightness distribution variations, most probably caused by strong flickering. Timedependent accretion disc self-occultation or variations of the hot spot(s) intensity are also possible explanations. Negative superhumps with period of $\sim 0 \mathrm{~d} .145$ are detected in two long runs in 2000. A possible connection between SW Sex and negative superhump phenomena through the presence of tilted accretion disc is discussed, and a way to observationally test this is suggested.
\end{abstract}

Key words. accretion, accretion discs - stars: binaries: eclipsing - stars: individual: BH Lyn - stars: novae, cataclysmic variables

\section{Introduction}

BH Lyncis is an eclipsing novalike (NL) cataclysmic variable (CV) with an orbital period of $\sim 3$ h.74 (Andronov et al. 1989). Thorstensen et al. (1991a), Dhillon et al. (1992), and Hoard \& Szkody (1997) have shown that spectral behavior of BH Lyn resembles that of SW Sex-type novalikes. SW Sex stars are spectroscopically defined sub-class of novalikes (Thorstensen et al. 1991b). Most of them are eclipsing, but show single-peaked emission lines contrary to the expected double-peaked from high-inclined accretion discs (ADs). Other distinctive characteristics are high-velocity emission components, narrow absorption components superimposed over emission lines around orbital phase 0.5 , and a large phase offset of the emission line radial velocities, with respect to the photometric conjunction. The eclipse profiles are V-shaped rather that U-shaped, and the accretion discs brightness temperature distribution derived from eclipse mapping is much flatter than expected for a steady-state accretion disc (e.g. Rutten et al. 1992). Patterson (1999) reports that most of the SW Sex stars show both negative and positive superhumps. Besides, some of the members show low states (Honeycutt et al. 1993). Currently, there is no widely accepted model of SW Sex stars. In most of the CVs, the accretions stream from secondary hits on the outer disc edge, and a hot spot is formed at the impact. In the most elaborated model of the SW Sex stars, Hellier (1998) suggested that part of the gas in the stream does not stop in the vicinity of the hot spot. Instead, it continues moving above the disc surface, hits the disc close to the white dwarf, and thus forms a second spot. Recently, Rodriguez-Gil et al. (2001) discovered variable circular

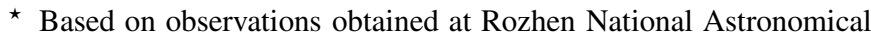
Observatory, Bulgaria. polarization in LS Peg and suggested that SW Sex stars are intermediate polars with the highest mass accretion rates.

The object of this study, BH Lyn, is mostly studied spectroscopically, and the existing photometric data are generally used to obtain the eclipse ephemeris and to supplement the spectral observations. In this paper, we report the results of our photometry of BH Lyn obtained in 1999-2004.

\section{Observations and data reduction}

The photometric observations of BH Lyn were obtained with the 2.0-m telescope in the Rozhen Observatory. A Photometrics $1024^{2}$ CCD camera and a Johnson $V$ filter were used. The CCD camera was $2 \times 2$ pixels binned, which resulted in $\sim 13 \mathrm{~s}$ of read-out dead-time. In total, 7 runs of photometric data were obtained between 1999 and 2004. The exposure time used was between 30 and $60 \mathrm{~s}$. Some details of the observations are given in Table 1. After bias and flat-field corrections, the photometry was done with the standard DAOPHOT aperture photometry procedures (Stetson 1987). The magnitude of BH Lyn was measured relative to the star BH Lyn-5 $(V=14.47)$, and BH Lyn-4 $(V=15.30)$ served as a check (Henden \& Honeycutt 1995). The runs are shown in Fig. 1, and it appears that BH Lyn was in high state during all observations.

\section{Results}

The eclipse timings given in Table 1 were determined by fitting a parabola to the lower half of the eclipses. To refine the orbital ephemeris of BH Lyn, we also used the eclipse timings collected by Hoard \& Szkody (1997). The O-C residuals with respect to the best linear ephemeris are shown in Fig. 2. Clearly, 


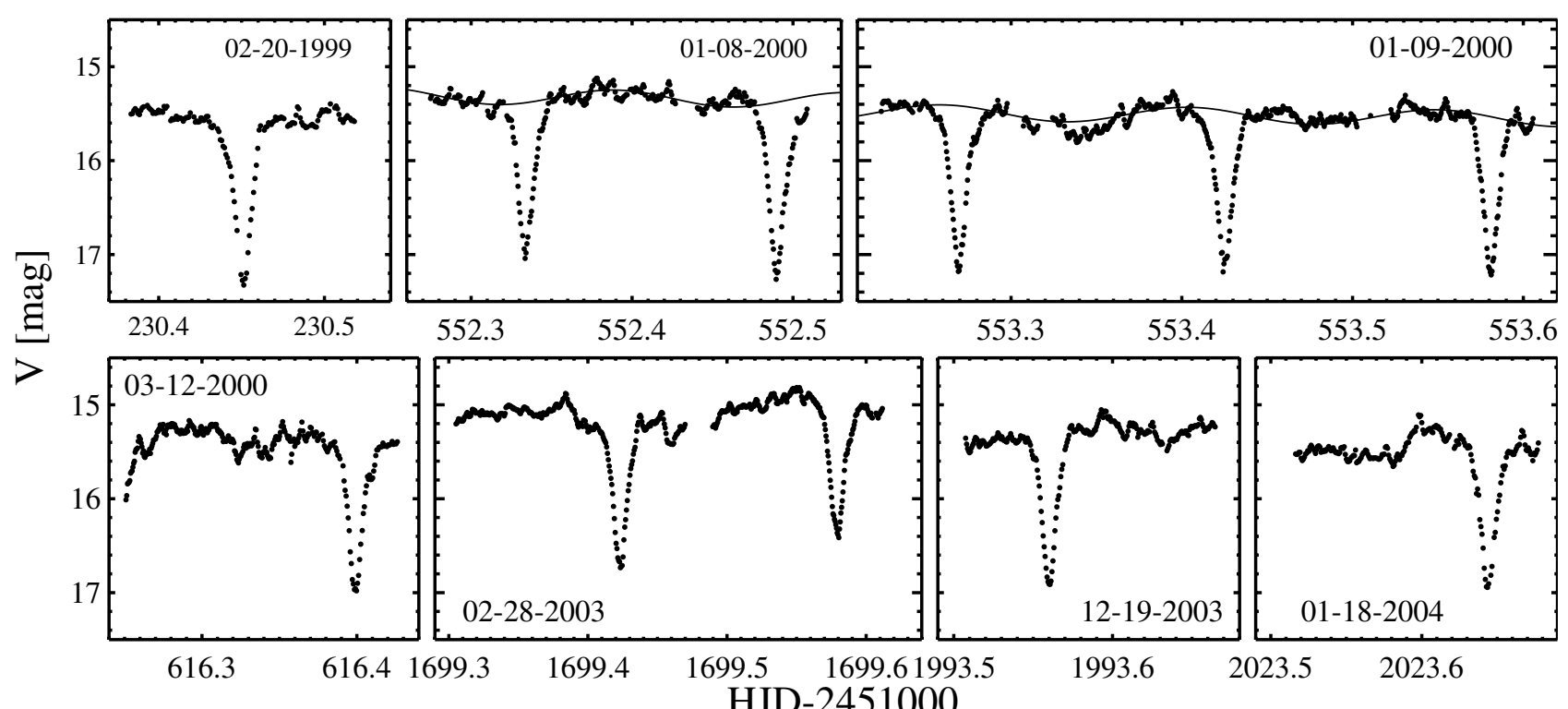

Fig. 1. $V$-band observations of BH Lyn. The solid line shows the sinusoidal fit with the period of the superhumps detected in the 2000 data.

Table 1. $V$ band observations of BH Lyn. The eclipse timings are also given.

\begin{tabular}{cccc}
\hline \hline UT date & $\begin{array}{c}\text { HJD Start } \\
-2451000\end{array}$ & $\begin{array}{c}\text { Duration } \\
{[\mathrm{h}]}\end{array}$ & $\begin{array}{c}\text { HJD mid-eclipse } \\
-2451000\end{array}$ \\
\hline Feb. 20, 1999 & 230.3833 & 3.23 & 230.45114 \\
Jan. 08, 2000 & 552.2748 & 5.61 & 552.33385 \\
& & & 552.48983 \\
Jan. 09, 2000 & 553.2240 & 9.16 & 553.26945 \\
& & & 553.42513 \\
& & & 553.58089 \\
Mar. 12, 2000 & 616.2509 & 4.21 & 616.39900 \\
Feb. 28, 2003 & 1699.3052 & 7.44 & 1699.42318 \\
& & & 1699.57916 \\
Dec. 19, 2003 & 1993.5075 & 3.77 & 1993.56007 \\
Jan. 18, 2004 & 2023.5165 & 3.86 & 2023.64410 \\
\hline
\end{tabular}

the linear ephemeris does not describe the eclipse times well and, as Hoard \& Szkody (1997) point out, this is mainly due to the anomalously large, positive residual of the first eclipse timing. Hoard \& Szkody (1997) suggested that the first eclipse timing was in error and calculated a linear ephemeris without it. The $\mathrm{O}-\mathrm{C}$ residuals of our new eclipse timings are rather large, $\sim 0$ d.006, and increasingly positive. Together with the first two timings, whose $\mathrm{O}-\mathrm{C}$ residuals are also positive, this suggests the presence of a curvature in the $\mathrm{O}-\mathrm{C}$ residuals. The dashed line is the second-order polynomial fit to all eclipse timings. The quadratic term is $7.6 \times 10^{-12}$ and implies that the orbital period of BH Lyn increases on a time scale of $\sim 4.2 \times 10^{6} \mathrm{yr}$. In most of the CVs, the mass donor star is the less massive one, and hence, if the mass transfer is conservative, the orbital period of the system will increase. For plausible component masses in BH Lyn, $M_{\mathrm{WD}} \sim 0.73$ and $M_{2} \sim 0.33$ (Hoard \& Szkody 1997), the mass transfer rate should be $\dot{M} \sim 5 \times 10^{-8} M_{\odot} \mathrm{yr}^{-1}$ to be compatible with the putative orbital period increase. However, there are several arguments against this scenario. First, there is a bulk of evidence that CVs evolve toward shorter orbital periods due to the angular momentum loss of the secondary by magnetic breaking (Warner 1995). Second, $\dot{M} \sim 5 \times 10^{-8} M_{\odot} \mathrm{yr}^{-1}$ is probably too high and generally not typical for CVs. Third, the eclipse timings presented by Andronov et al. (1989) have been determined

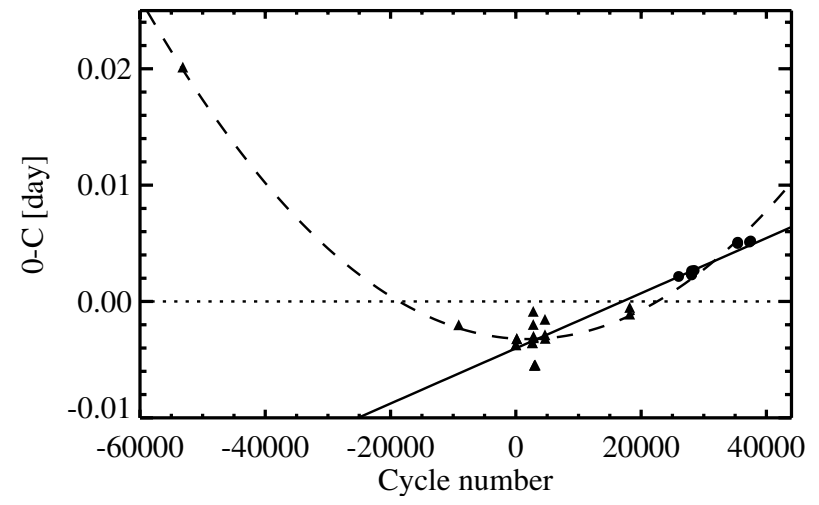

Fig. 2. O-C residuals of the minima with respect to the best linear ephemeris. The second-order polynomial fit to the $\mathrm{O}-\mathrm{C}$ residuals is also shown. The solid line is our best linear ephemeris. The filled circles show our new timings.

by the phase folding of observations with photographic plates with rather long exposure times of 8,12 , and $30 \mathrm{~min}$. It is not surprising then, that those timings exhibit relatively large scatter (the timings with cycle numbers $~ 3000$ ). The second timing has been determined from plates with exposure time $30 \mathrm{~min}$, only slightly shorter than the total eclipse duration, and the large positive $\mathrm{O}-\mathrm{C}$ of this timing may be a statistical fluctuation. Because the first two timings are the ones that determine the curvature in the $\mathrm{O}-\mathrm{C}$ residuals, one may question whether the curvature is real. Future observations may prove that the orbital period of $\mathrm{BH}$ Lyn increases, however, our opinion is that only two timings determined from patrol plates do not provide enough evidence for this. We therefore determined an updated linear ephemeris without using the first two timings:

$\mathrm{HJD}_{\min }=2447180.33600(28)+0.155875577(14) E$.

This ephemeris is shown by the solid line in Fig. 2. It is very similar to the ephemeris of Hoard \& Szkody (1997); the orbital period is only slightly larger and the reference times differ by $\leq 1 \mathrm{~min}$.

The light curves show prominent humps whose maxima occur at different orbital phases in the different runs (Fig. 1). We 


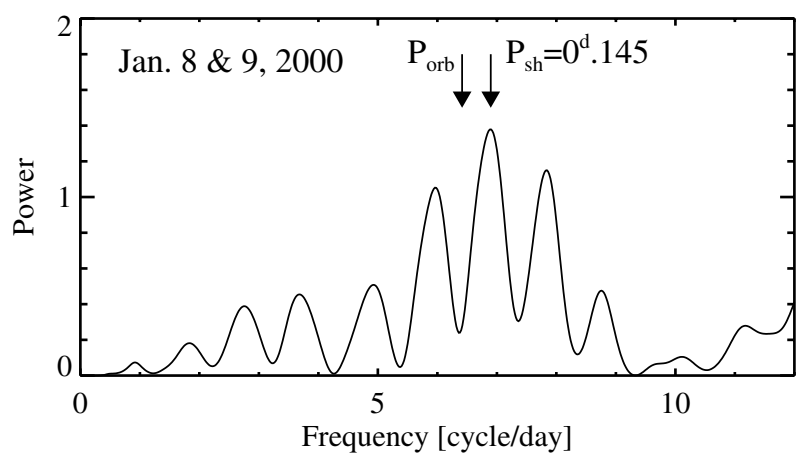

Fig. 3. Periodogram of the January 2000 data. The negative superhump and the orbital periods are indicated.

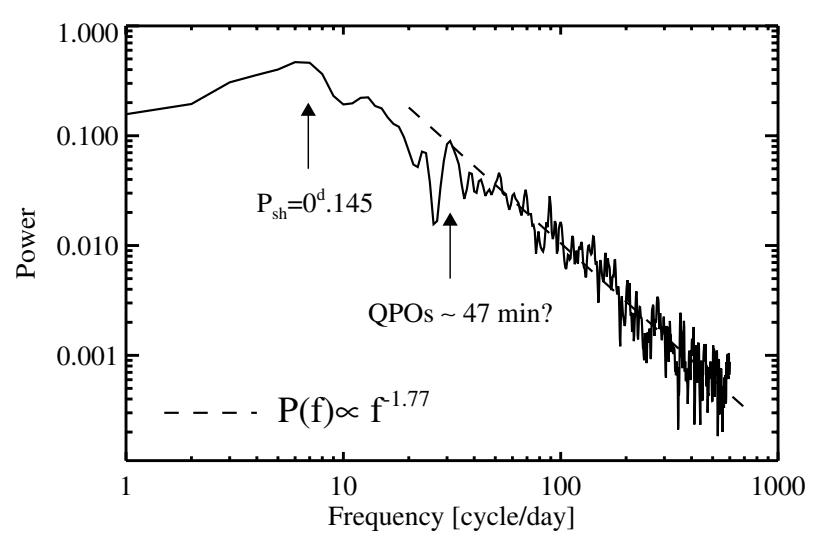

Fig. 4. The mean power spectrum of BH Lyn light curves.

interpret this as an indication of the presence of superhumps. Because our data are sparse, they are clearly not enough for an in-depth study of superhumps in BH Lyn. After removing the data during eclipses, we computed the Lomb-Scargle periodogram (Scargle 1982) of the two January 2000 series only (Fig. 3). The strongest peak around the expected frequency of the superhumps corresponds to a period of $\sim 0 \mathrm{~d} 1450 \pm 0.0065$, which is close to the negative superhumps period $0.1490 \pm 0.0011$ reported by Patterson (1999). The least-squares fit gives the semiamplitude of the signal of $0.084 \pm 0.005 \mathrm{mag}$.

We have also searched all runs for periodic variations on the minute time-scale. The power spectra show many peaks with frequencies below $\sim 150{\text { cycle } \mathrm{day}^{-1}}^{-1}$, but the attempts to fit the runs with periods corresponding to any of the peaks in the periodograms were not satisfactory. Thus, most probably no coherent oscillations are present. The individual power spectra show a typical red noise shape characterized by a power-law decrease of the power with frequency $P(f)=f^{\gamma}$. The mean power spectrum of BH Lyn has power-law index $\gamma=-1.77$. Because the red noise processes have strong low-frequency variability, it is most likely that the peaks in the periodograms are due to the red noise. Nevertheless, the peak at $\sim 32$ cycles day $^{-1}$ is present in most periodograms, and it is also noticeable in the mean power spectrum (Fig. 4). This might indicate the presence of quasi-periodic oscillations like the ones discussed by Patterson et al. (2002), however, a study based on more data is needed to confirm this.

The red noise in the power spectra of CVs is a result of flickering (Bruch 1992). BH Lyn light curves show strong flickering activity; flickering peaks with typical durations of 5-10 min and amplitudes reaching $\sim 0.2$ mag can be recognized in Fig. 1 . The mean standard deviation in the light curves after the lowfrequency signals have been subtracted is $\sim 0.06 \mathrm{mag}$. This value

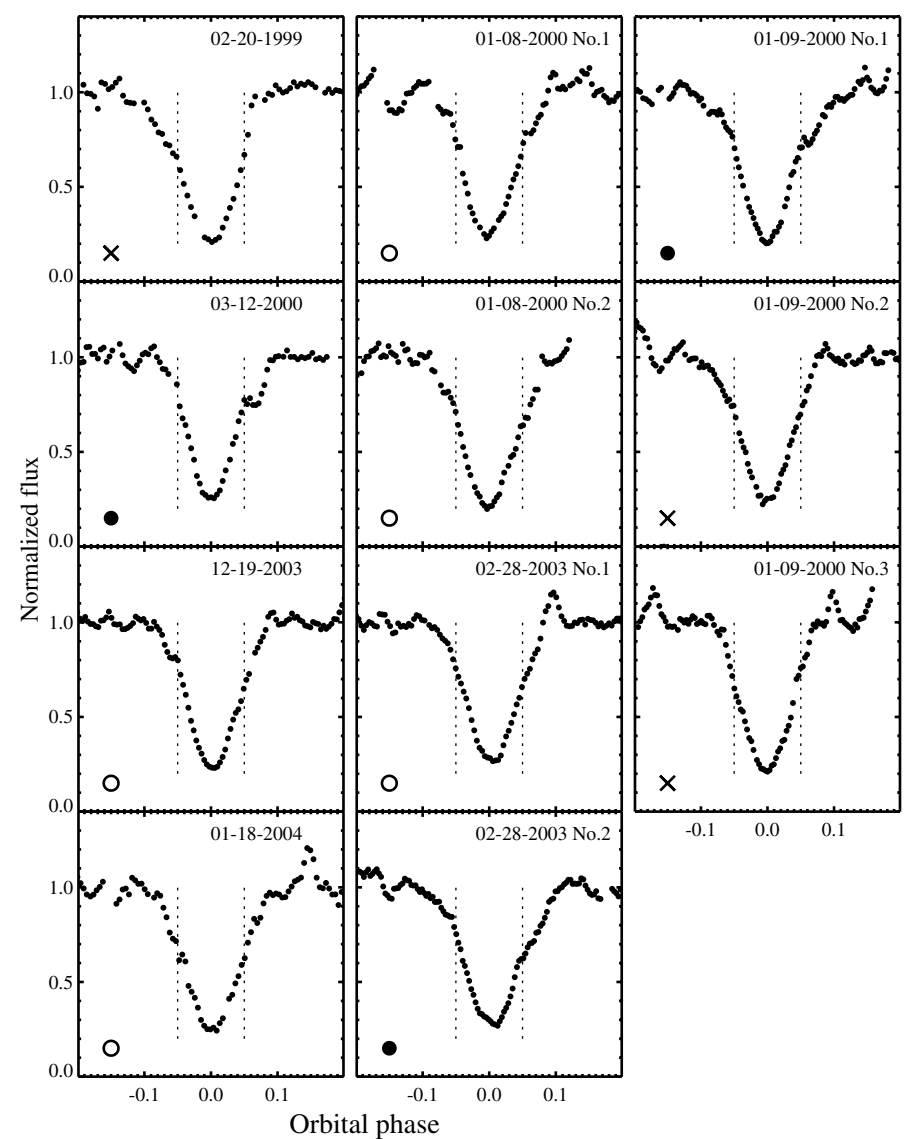

Fig. 5. Normalized eclipses of BH Lyn. The dashed lines are guide to the eye to see the difference of the eclipse profile easier. The symbols used for the eclipses in Fig. 6 are shown in the lower left corners.

is consistent with the standard deviation found in the light curves of the NLs TT Ari, MV Lyr and PX And (Kraicheva et al. 1999a,b; Stanishev et al. 2002).

The depth of the eclipses in BH Lyn during our observations is $\sim 1.5 \mathrm{mag}$, and their average full-width at half-flux is $0.0683( \pm 0.0054) P_{\text {orb }}$. The out-of-eclipse magnitudes were fitted with low-order polynomial functions to account for brightness variations that are not due to the eclipse, but most probably arise from the superhumps. The eclipses were normalized to the fits and are shown in Fig. 5. As can be seen, there is a substantial variability of the eclipse shape, even during a single night. The variations are most notable in the upper half of the eclipse profiles. Half of the eclipses appear to be fairly symmetric, while the rest are clearly asymmetric. More interestingly, though, the egress of the eclipses on Mar. 12, 2000 and No. 1 on Jan. 9, 2000 , and possibly the ingress of some other eclipses, are not monotonic. To highlight the differences, in Fig. 6 we show all the eclipses together. Except for the single eclipse in 1999, the ingress of all eclipses are very similar. The egress of the eclipse are however very different, and the eclipses could be split into three sequences. In Fig. 6, each of these groups is plotted with a different symbol.

\section{Discussion}

Because of the large variability of the eclipse profiles in BH Lyn, we are reluctant to attempt eclipse mapping or to try to estimate the system parameters from the eclipse width. Clearly, such analyzes could give false results. The rather rapid changes in the 


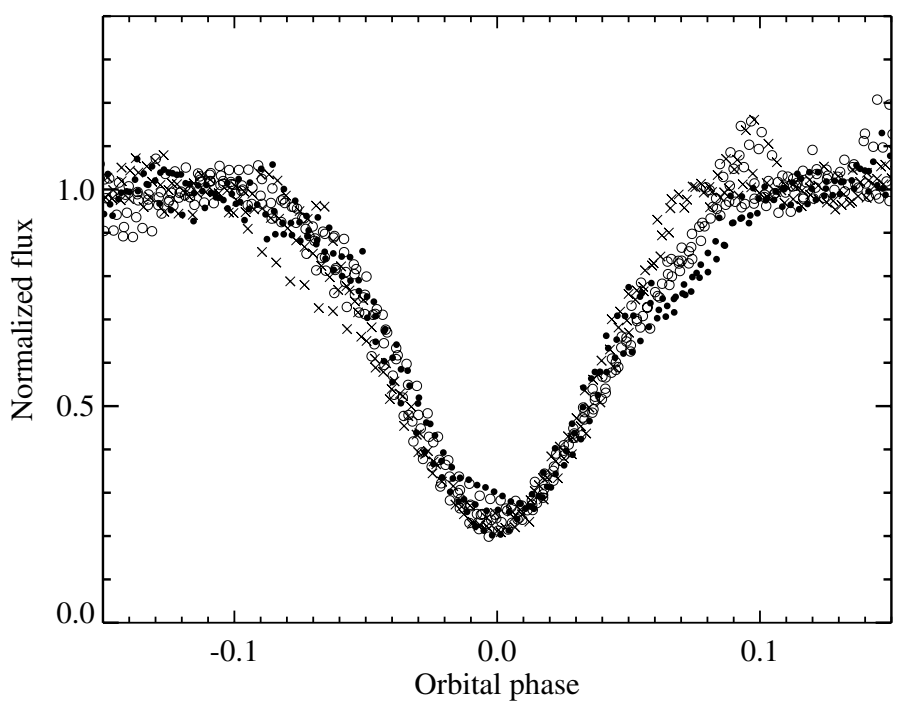

Fig. 6. The three eclipse groups plotted together with different symbols.

eclipse profiles, even during a single night, could be explained by temporal variations of the $\mathrm{AD}$ surface brightness distribution. Large flickering peaks can be seen before or after some of the eclipses (Fig. 5). If such a peak occurs during an eclipse, it could alter its shape, even to cause the eclipse not to be monotonic. Another explanation could be that the amount of overflowing gas varies, and as a consequence the intensity of the two hot spots could also change, causing variations in the eclipse profile.

Variations of the area of the eclipsing body with time will also cause variations of the eclipses. Given the time scale of the observed changes, the secondary is ruled out. On the other hand, the SW Sex stars most likely possess very complex accretion structures, and it may be that the $\mathrm{AD}$ is self-occulting. Self-occultation seems to be the most reasonable explanation of the UV observations of another SW Sex star, DW UMa (Knigge et al. 2000), hence giving support for this in BH Lyn. Variations of the effective area of the occulting parts may cause the observed eclipse profile changes.

The presence of negative superhumps in eclipsing SW Sex stars is very interesting. The origin of negative superhumps is still a puzzle, but they are believed to be caused by a retrograde precession of an accretion disc that is tilted with respect to the orbital plane (Bonnet-Bidaud et al. 1985). If negative superhumps do arise from the precession of tilted ADs, then the accretion stream overflow would easily occur (Patterson et al. 1997). Therefore, the SW Sex and negative superhumps phenomena should have the same origin. Due to the presence of precessing tilted $\mathrm{AD}$, the amount of gas in the overflowing stream will be modulated on the negative superhump period. Hence, the intensity of the second hot spot will change and may produce superhumps (Patterson et al. 1997; Stanishev et al. 2002). This scenario can be observationally tested. In this model, the negative superhumps should manifest themselves in spectra in two ways: 1) the intensity of the high-velocity emission components in spectra, which are thought to arise from the second spot, should be modulated with the superhumps period; 2) since the orientation of the tilted disc with respect to the observer will change over the precession cycle, at certain precession phases, the SW Sex signatures should disappear. To test these predictions, time-resolved high signal-to-noise spectrophotometry over several consecutive nights is needed, since the precession periods are of the order of a few days. We encourage such studies.

Acknowledgements. The work was partially supported by NFSR under project No. 715/97.

\section{References}

Andronov, I. L., Kimeridze, G. N., Richter, G. A., \& Smykov, V. P. 1989, IBVS, 3388

Bonnet-Bidaud, J. M., Motch, C., \& Mouchet, M. 1985, A\&A, 143, 313

Bruch, A. 1992, A\&A, 266, 237

Dhillon, V. S., Jones, D. H., Marsh, T. R., \& Smith, R. C. 1992, MNRAS, 258, 225

Hellier, C. 1998, PASP, 110, 420

Henden, A. A., \& Honeycutt, R. K. 1995, PASP, 107, 324

Hoard, D. W., \& Szkody, 1997, ApJ, 481, 433

Honeycutt, R. K., Livio, M., \& Robertson, J. W. 1993, PASP, 105, 922

Knigge, C., Long, K. S., Hoard, D. W., Szkody, P., \& Dhillon, V. S. 2000, ApJ, 539, L49

Kraicheva, Z., Stanishev, V., Genkov, V., \& Iliev, L. 1999a, A\&A, 351, 607

Kraicheva, Z., Stanishev, V., \& Genkov, V. 1999b, A\&AS, 134, 263

Patterson, J. 1999, in Disk Instabilities in Close Binary Systems, ed. S. Mineshige, \& J. C. Wheeler (Tokyo: Universal Academy Press), 61 Patterson, J., Kemp, J., Saad, J., et al. 1997, PASP, 109, 468

Patterson, J., Thorstensen, J. R., Kemp, J., et al. 2002, PASP, 114, 1364

Rodriguez-Gil, P., Casares, J., Martinez-Pais, I. G., Hakala, P., \& Steeghs, D. 2001, ApJ, 548, L49

Rutten, R. G. M., van Paradijs, J., \& Tinbergen, J. 1992, A\&A, 260, 213

Scargle, J. D. 1982, ApJ, 263, 835

Stanishev, V., Kraicheva, Z., Boffin, H., \& Genkov, V. 2002, A\&A, 394, 625

Stanishev, V., Kraicheva, Z., Boffin, H. M. J., et al. 2004, A\&A, 416, 1057

Stetson, P. 1987, PASP, 99, 191

Thorstensen, J. R., Davis, M. K., \& Ringwald, F. A. 1991a, ApJ, 327, 248

Thorstensen, J. R., Ringwald, F. A., Wade, R. A., Schmidt, G. D., \& Norsworthy, J. E. 1991b, AJ, 102, 272

Warner, B. 1995, The cataclysmic variables stars (Cambridge: Cambridge University Press) 